\title{
Heritage Resources as a Method to Reviving the Identity of Contemporary Interior Designs
}

\section{A Comparative Analysis of Users' Preferences of Interior Space}

\author{
DOI: $10.21625 /$ archive.v1i1.109 \\ Hala Barakat Elnaggar ${ }^{1}$ \\ ${ }^{1}$ Arab Academy for Science, Technology \& Maritime Transport, Cairo, Egypt
}

\section{Keywords}

Elements, Heritage, Identity, Culture.

\begin{abstract}
Egyptian heritage is known to be a result of many great civilizations. With various traditional elements and special features that add prominence to its cultural aspects, it carries magnificent aesthetic values and visual forms. Nonetheless, and due to the different nature of the cities and provinces in Egypt, styles and features in each region have taken different paths. For instance, some areas were influenced by Ancient Egyptians while others were more affected by Islamic or Coptic civilizations. Some regions were preserved in Nubian folk art heritage form. In the past, the country had a clear and unique identity that reflected its characteristics, environmental benefits and socio-cultural attributes. However, today the identity is faded and is nearly completely wiped by Western notions erasing our ideas, identities, and thoughts. This study focuses on the elements of heritage, their impact on people and the way these elements inspire interior architecture, form and psychology.
\end{abstract}

This study aims to discern the elements of heritage and identify the character and special criteria of each civilization such as the Ancient Egyptian, Islamic and Nubian folk art heritage with special references and clarifications as to the criteria of reviving the traditional identity in contemporary interior design. This study will also include an anlaysis of user preferences in relation to discussed features.

\section{Introduction}

The Egyptian society suffered since the beginning of the Renaissance period and until the end of the last century from multi-cultural fluctuations that imposed different cultural realities and caused changes in its culture. Consequently, there emerged a series of architectural identities that has experienced changes over time. Interior design is a field that does not deviate from the traditional in a time the contemporary is known to have quickly achieved uniqueness and creativity. However, the interior designer attempts to express historical dimensions, heritage and cultural privacy distinctively, especially when cultural boundaries seem to no longer exist. Thus, a need to stick to heritage and one's original identity has become essential. Therefore, the designer must understand the philosophical dimensions and concepts of sustainability sustaining our heritage. Here, one we must refer to the importance of emphasizing to the designer the need to remain consistent with features of the traditional in the formation of design solutions and to create a balance between heritage and modernity. The designer must also 
focus on reshaping the traditional to cope with the contemporary's functional needs as to create forms and designs suitable to our present time.

\section{1-1 Research problem}

Although the identity and use of traditional elements, both, possess significance, some negligence in considering these items and their characteristics, selecting appropriate concepts and architectural principles, and working on the formulation to achieve a proper balance between the heritage and the contemporary has been noted. There is a need to rearticulating and recreating an understanding of heritage to meet such functional needs and an emphasis on the intellectual identity. The expected result then would be a production of a kind of architecture that parallels the spirit of the age and its cultural identity within residential areas.

\section{1-2 Goal and Approach}

The research aims to focus on the importance of preserving the identity and heritage through elements of the internal environment to:

1. Find the standards and objectives that affect the identity of contemporary Egyptian interior architecture.

2. Emphasize the importance of identity within the interior design of a house.

3. Link contemporary and the traditional identity by locating contemporary interior architecture in interior design.

4. Reveal the identity and artistic value that accompanies heritage.

\subsection{Research Methodology}

The research starts with a theoretical study to identify the semantic, intellectual and artistic work standards as well as elements that facilitate the formation of an identity. The study discusses the fundamental concepts of ancient Egyptian designs such the Islamic and the Nubian. It also highlights the designing processes which are inspired from and filled with the mentioned elements. Henceforth, explanations, ideas and solutions are formed about the possibility of retaining, or rather re-using, these elements and architectural characteristics. Furthermore, a questionnaire about the local traditional style preferences and modern European-styles will be analyzed. Following would be a design questionnaire in which the contemporary elements and the principles are engaged using modern techniques.

\section{LITERATURE REVIEW}

Housing preferences have been studied from different theoretical perspectives. These studies can be classified to the following topics: the impact of sustainability trends in housing design and the identity of Arab cities (Rihan, 2012), The identity and cultural impact on the individual's behavior within architectural spaces (Azouz,2014), the Sustainable Application of Interior Spaces in Traditional Houses of the United Arab Emirates (Alkhalidi, 2012), the Revival of the Islamic Architectural Vocabulary in Jordan: A Case Study on the Use of Courtyards in Residential and Public Buildings (Abo-Ghanima, 2015), Place Identity of Residential Environment in Iraqi Contemporary Architectural Trends and its Impact on the Academic Architectural Product (Mumtaz, 2010), and Interior Design of Egyptian Residences between originality and modernity (Mashhour, 2011).

\section{METHODS}

\subsection{The Study Sample}

In The following analytical case study, a sample consisting of different age categories, 50, 40, 30, and 20, was selected and composed of almost a number of twenty people from each age group and various fields. The subjects consisted of both sexes, males and females, who are highly-qualified and have similar economic and social circumstances. The program's implementation lasted a week.

In the following questionnaire, the design process, aesthetic heritage elements, and different patterns of contemporary furniture and interior design were all taken into account. 


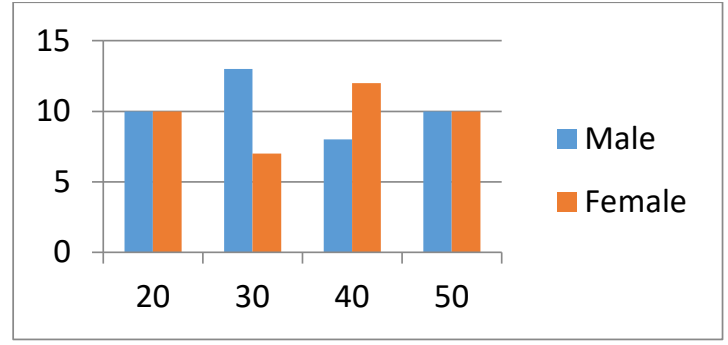

Figure.1 A display of the number of males and females of each age group.

\subsection{General Questionnaire Results}

The first questionnaire is a focused study of people's preferences with regards to traditional and modern European styles in interior spaces. The survey indicates that the different age groups preferred different styles. The fifty year old category preferred the local, or the traditional, with a percentage of 65 while $15 \%$ preferred to use modern European styles. The forty year old category equally enjoyed both styles. The thirty year old category had a preference for local traditional patterns by $45 \%$ as the modern European patterns ratio exceeded $35 \%$. As for the twenty year old category, the preference for local tradition patterns went down to $40 \%$ while their preference for the modern reached 55\%.

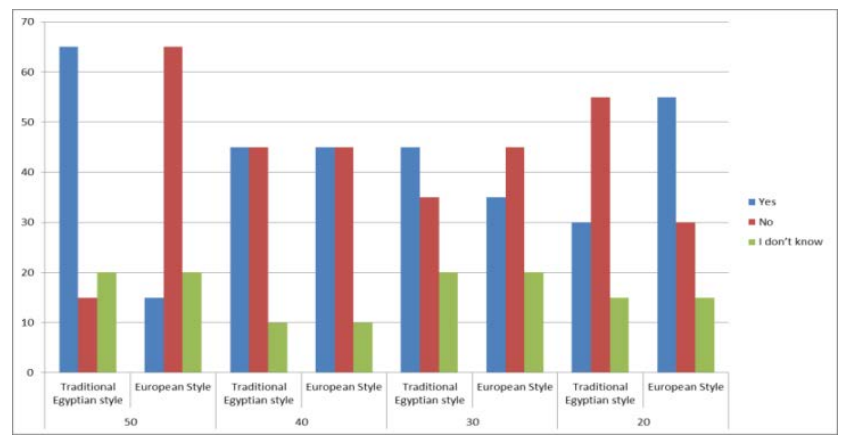

Figure.2 Comparative analysis of users' preference of interior spaces (European Style - Traditional Egyptian style)

Moreover, when asked about the reason for the absence of the traditional local models in home designing, the main reason was the absence of the appropriate traditional designs, high costs, and lack of quality and contemporary products that are suitable for the current internal space requirements. In the second phase, the questionnaire was presented in the form of images of designs with a modern style and a traditional cultural identity (ancient Egyptian - Islamic - Nubian) using relevant elements in the design of the walls, furniture, accessories, lighting and openings.

The questionnaire focused on the design, the process of organizing the visual elements including the methods, heritage expressions, and the impact or significance on the basis of design, vacuum form, color, surface textures, proportions, repetition, rhythm and elements of decoration. The design was simple, of quality and identity in an appealing manner and did not cause disruptions to the elements of design.

The questionnaire has started out by presenting a range of images for each civilization including the ancient Egyptian, Islamic, Nubian. Respondents were asked about the measure of their approval, as presented in an image below, of the aforementioned elements of heritage such as color, proportion, identity, decorative elements and repetition.

\begin{tabular}{|c|c|c|c|c|c|c|c|c|c|c|c|c|c|c|c|c|c|c|c|c|c|c|}
\hline \multicolumn{3}{|c|}{ Repetition } & \multicolumn{3}{|c|}{ Design pattem } & \multicolumn{3}{|c|}{ Identity } & \multicolumn{4}{|c|}{ Rhythm } & \multicolumn{3}{|c|}{ Proportion } & \multicolumn{3}{|c|}{ Colors } & \multicolumn{3}{|c|}{ Symbol } & $\begin{array}{l}\text { Evaluate } \\
\text { Picture }\end{array}$ \\
\hline
\end{tabular}

After clarifying and analyzing the images that were shown to the participants, as shown in the figure above, a proportion of the respondents tended to answer with "very good”, others with "good", and a few with "I don't know”.

\subsection{First questionnaire: The Identity of Heritage - Ancient Egypt}



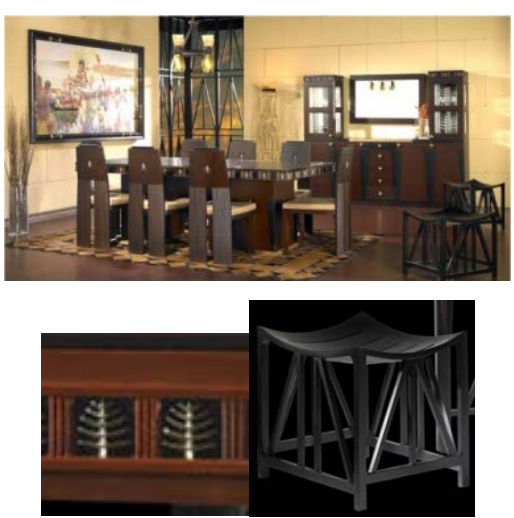
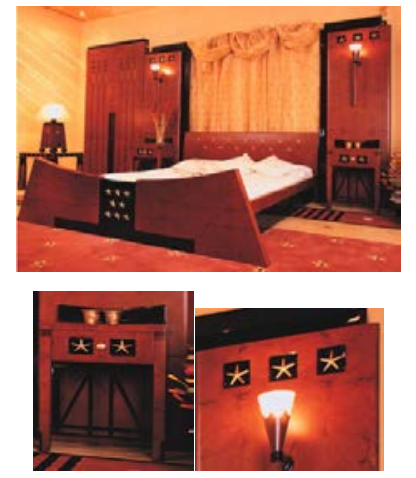
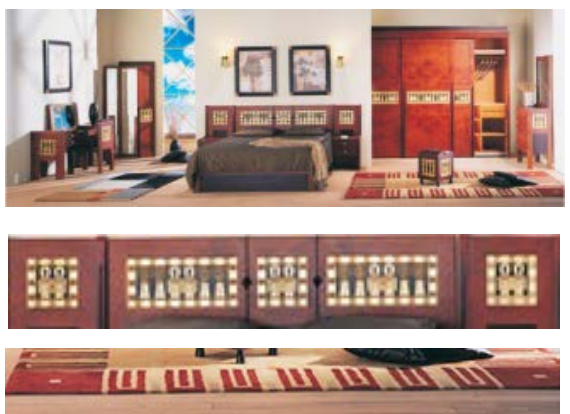

Fig.3 Hieroglyph \& symbolic art (left), Hieroglyph \& symbolic art (center), symbolic patterns (right). It has been clear from the questionnaire, as shown on the left of Figure 3 (left), that designs of ancient Egyptian style with a hint of modernism were seen by an average of $74 \%$ of the participants as very well-designed and appropriate in terms of use of elements and colors, proportion, rhythm, identity, the decorative elements and repetition. It was noted to satisfy their functional needs and provides them with beauty and identity in the design. Some believed it made them feel comfortable while $20 \%$ saw it as appropriately designed. Six percent were neutral and $70 \%$ of the participants were influenced and thought of changing spaces within their homes with similar designs.

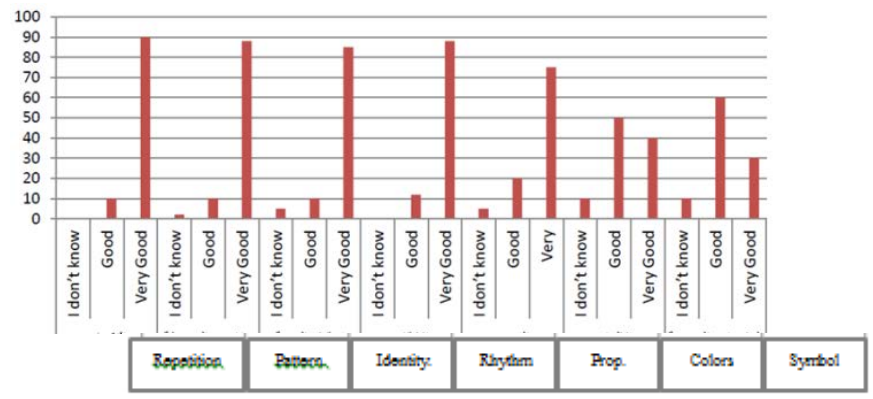

Fig.4 Average of Ancient Egyptian Design preferences (left)

It has been clear from the questionnaire, as shown in Fig 3 (center), that designs of ancient Egyptian style with a hint of modernism were seen by an average of $78 \%$ of the participants as very well-designed and appropriate in terms of use of elements and colors, proportion, rhythm, identity, the decorative elements and repetition. It was noted to satisfy their functional needs and provides them with beauty and identity in the design. Some believed it made them feel comfortable while $18 \%$ saw it was appropriately designed. Four percent were neutral and $75 \%$ of the participants were influenced and thought of changing spaces within their homes with similar designs.

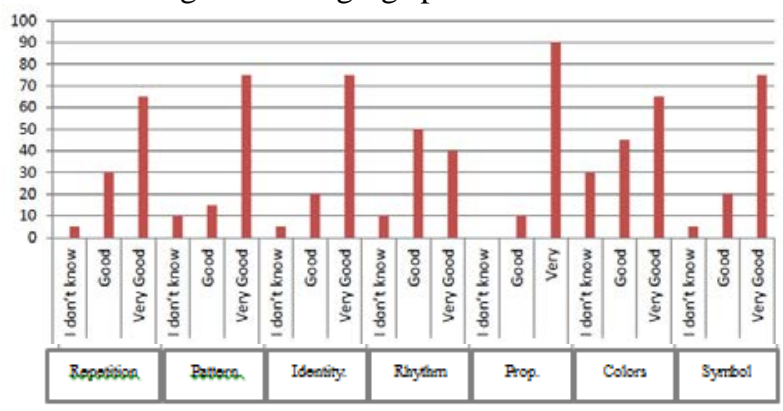

Fig.5 Average of Ancient Egyptian Design preferences (center)

It has been clear from the questionnaire, as shown in Fig 3 (right), that designs of ancient Egyptian style with a hint of modernism were seen by an average of $79 \%$ of the participants as very well-designed and appropriate in terms of use of elements and colors, proportion, rhythm, identity, the decorative elements and repetition. It was noted to satisfy their functional needs and provides them with beauty and identity in the design. Some believed it made them feel comfortable while $16 \%$ saw it was appropriately designed. Five percent were neutral and $70 \%$ of the participants were influenced and thought of changing spaces within their homes with similar designs. 


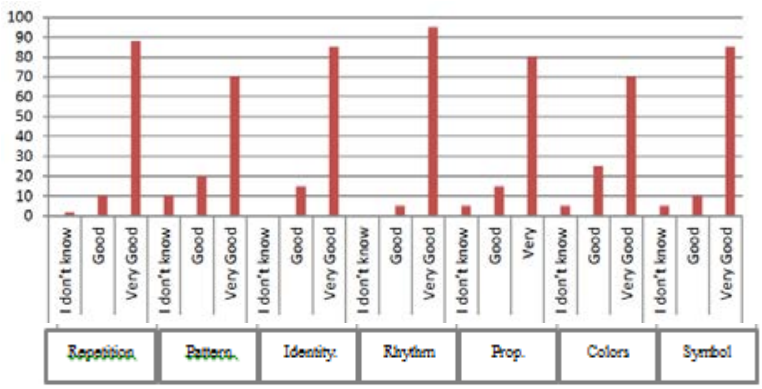

Fig.6 Average of Ancient Egyptian Design preferences (right)

\subsection{Second Questionnaire: The Identity of Heritage- the Islamic civilization}
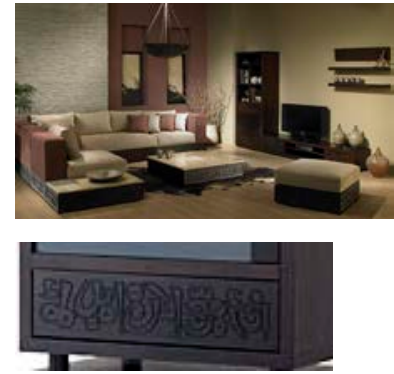
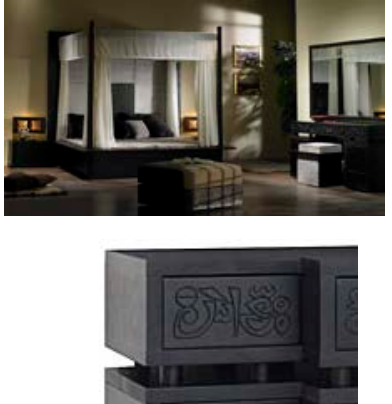
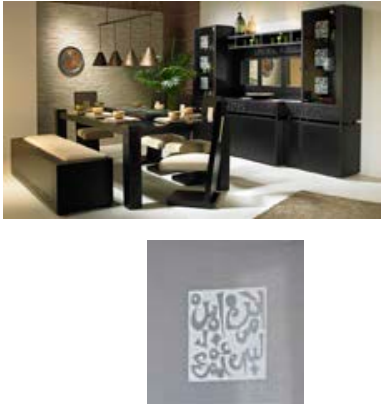

Fig.7 used of Calligraphy (left \& center) and symbolic patterns (right).

It has been clear from the questionnaire, as shown in Figure 7 (right), that designs Islamic styles with a hint of modernism were seen by an average of $69 \%$ of the participants as very well-designed and appropriate in terms of use of elements and colors, proportion, rhythm, identity, the decorative elements and repetition. It was noted to satisfy their functional needs and provides them with beauty and identity in the design. Some believed it made them feel comfortable while 23\% saw it was appropriately designed. Eight percent were neutral and 69\% of the participants were influenced and thought of changing spaces within their homes with similar designs.

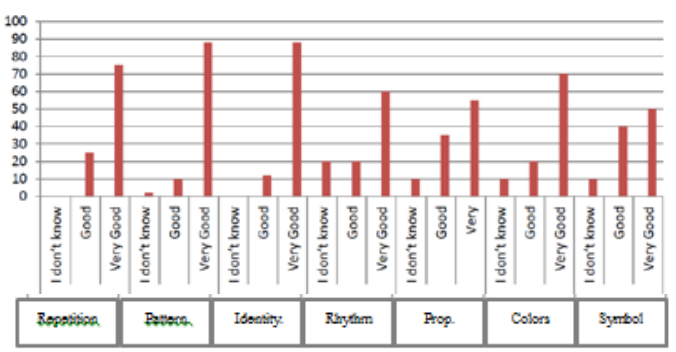

Fig.8 Average of Islamic Design preferences (left)

It has been clear from the questionnaire, as shown in Figure 7 (center), that designs of Islamic Styles with a hint of modernism were seen by an average of $80 \%$ of the participants as very well-designed and appropriate in terms of use of elements and colors, proportion, rhythm, identity, the decorative elements and repetition. It was noted to satisfy their functional needs and provides them with beauty and identity in the design. Some believed it made them feel comfortable. Seven percent were neutral and $73 \%$ of the participants were influenced and thought of changing spaces within their homes with similar designs.

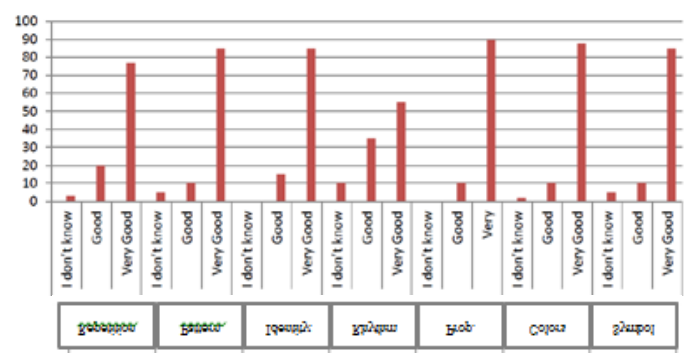

Fig.9 Average of Islamic Design preferences (center) 
It has been clear from the questionnaire, as shown in Fig 7 (right), that designs of Islamic Styles with a hint of modernism were seen by an average of $82 \%$ of the participants as very well-designed and appropriate in terms of use of elements and colors, proportion, rhythm, identity, the decorative elements and repetition. It was noted to satisfy their functional needs and provides them with beauty and identity in the design. Some believed it made them feel comfortable while $14 \%$ saw it was appropriately designed. Four percent were neutral and $80 \%$ of the participants were influenced and thought of changing spaces within their homes with similar designs.

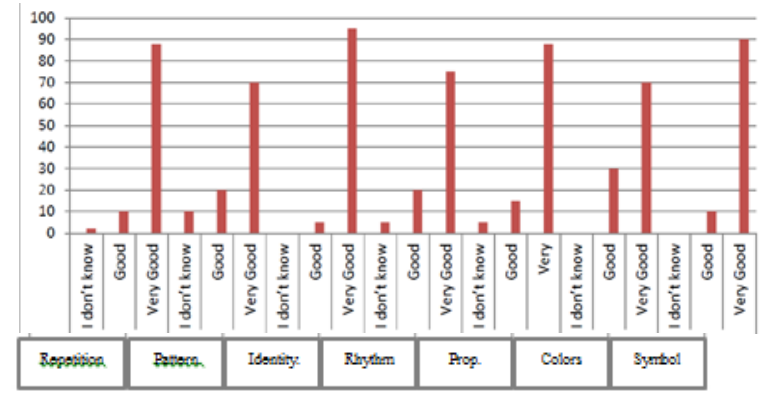

Fig.10 Average of Islamic Design preferences (right)

\subsection{Third Questionnaire: The Identity of Heritage - Nubian Heritage.}
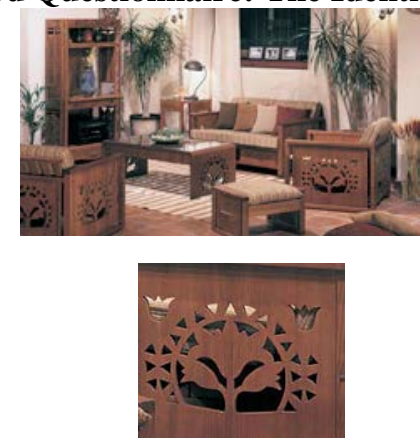
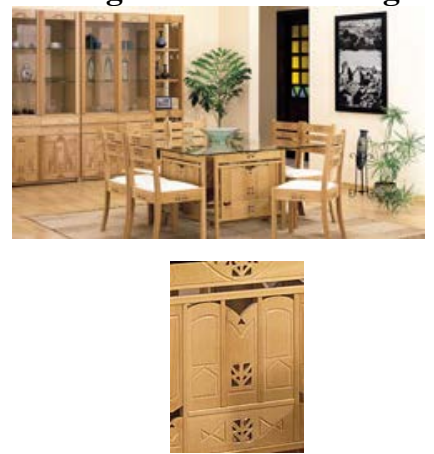
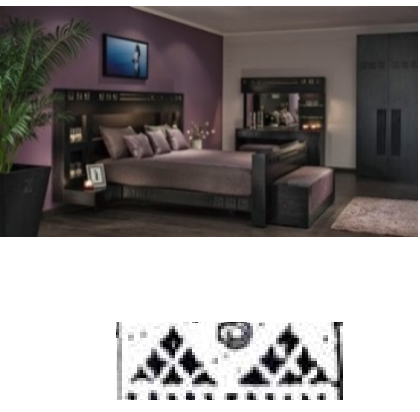

Fig.11 The lotiform flower (left), Symbolic Folk Arts (center \& right)

It has been clear from the questionnaire, as shown in Figure 11 (left), that designs of Nubian Styles with a hint of modernism were seen by an average of $70 \%$ of the participants as very well-designed and appropriate in terms of use of elements and colors, proportion, rhythm, identity, the decorative elements and repetition. It was noted to satisfy their functional needs and provides them with beauty and identity in the design. Some believed it made them feel comfortable while $25 \%$ saw it was appropriately designed. Five percent were neutral and $60 \%$ of the participants were influenced and thought of changing spaces within their homes with similar designs.

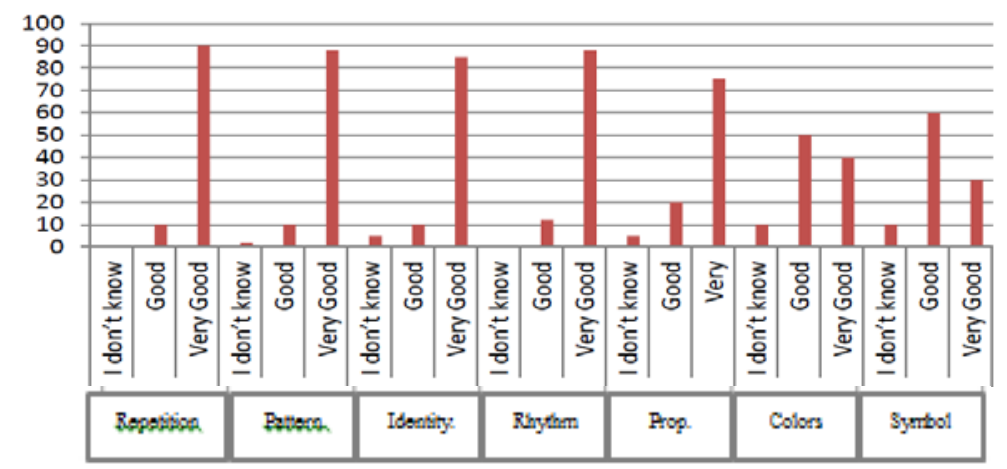

Fig.12 Average of Nubian Design preferences (left)

It has been clear from the questionnaire, as shown in Figure 11 (center), that designs of Nubian Styles with a hint of modernism were seen by an average of $69 \%$ of the participants as very well-designed and appropriate in terms of use of elements and colors, proportion, rhythm, identity, the decorative elements and repetition. It was noted to satisfy their functional needs and provides them with beauty and identity in the design. Some believed it made 
them feel comfortable while $27 \%$ saw it was appropriately designed. Four percent were neutral and $60 \%$ of the participants were influenced and thought of changing spaces within their homes with similar designs.

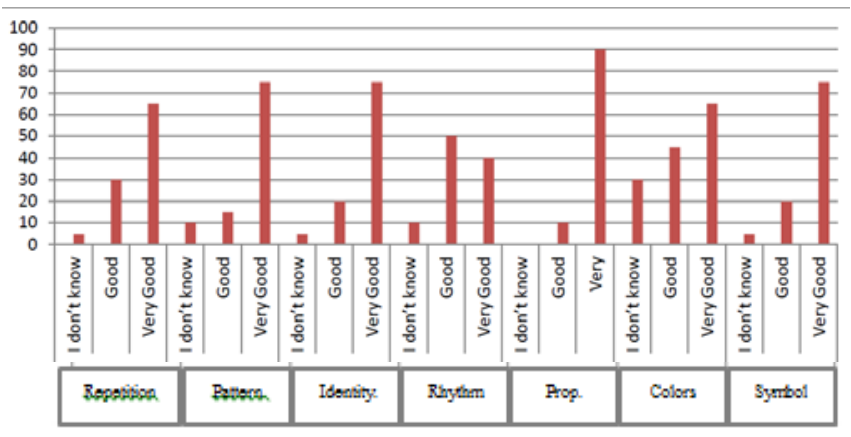

Fig.13 Average of Nubian Design preferences (center)

It has been clear from the questionnaire, as shown in Figure 11 (right), that designs of Nubian Styles with a hint of modernism were seen by an average of $81 \%$ of the participants as very well-designed and appropriate in terms of use of elements and colors, proportion, rhythm, identity, the decorative elements and repetition. It was noted to satisfy their functional needs and provides them with beauty and identity in the design. Some believed it made them feel comfortable while $14 \%$ saw it was appropriately designed. Five percent were neutral and $65 \%$ of the participants were influenced and thought of changing spaces within their homes with similar designs.

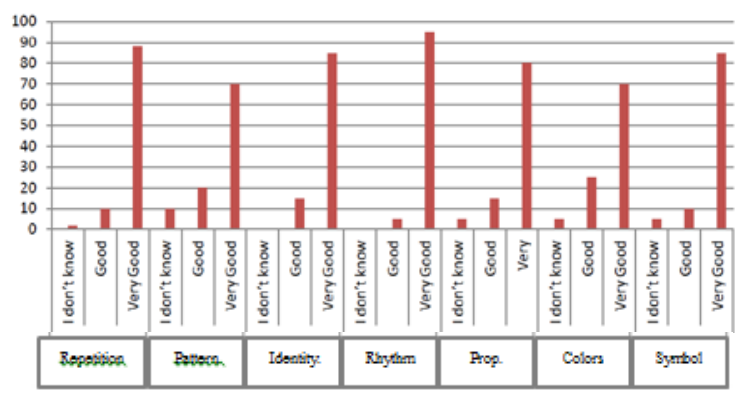

Fig.14 Average of Nubian Design preferences (right)

The results of the study in employing the definition of heritage within a space showed the inclination of the participants to simplicity and a combination of heritage values and contemporary design. The aims of the research included expressing the participants' identity through their design preferences inside their homes. Moreover, it showed how they were influenced by the ideas offered to them through the questionnaire.

\section{RESULTS}

The research study aims at identifying heritage concepts and achieving the relationship between tradition and modernity. The results of this study are as follows dictated that the materials and new technologies helped show local interior architecture with new solutions free from constraints. Heritage, as well, was identified with stability and continuity, thus, expressing a dire need of taking advantage of it in various ways and using it in today's designs. Dealing with heritage, especially the environmental aspects associated with it, and learning from past experiences in creating solutions to the current problems build a link between interior architectural heritage and contemporary designs. Moreover, dealing with heritage and integrating it with contemporary architecture leads to an understanding of heritage and an evaluation of requirements that match and reflect current times.

\section{RECOMMENDATIONS}

The research study is concluded to provide recommendations that should strengthen the vision the future vision of integrating heritage and contemporary interior architecture. These recommendations can be summarized as follows:

1. Working on strengthening the cultural awareness among members of the society as well as the importance of the local architectural heritage. Avoiding the blind imitation of the western thought and sticking to one's own. 
2. Engineers and architects are encouraged to develop the traditional elements to match current times after undertaking proper studies.

3. Confirming the inspiring use of patterns stemming from our heritage and its integration with design aspects that increase innovation revives the identity within the interior design elements.

4. Paying attention to the analysis of the patterns, traditions and cultures as a way of enriching design ideas.

\section{References}

1. Ali, W. (1999). The arab contribution to islamic art: From the seventh to the fifteenth centuries. Cairo, Egypt, Jordan, Royal Society of Fine Arts.

2. Alrashed, F., Asif, M., \& Burek, S. (2017). The Role of Vernacular Construction Techniques and Materials for Developing Zero-Energy Homes in Various Desert Climates. Buildings, 7(1), 17. doi:10.3390/buildings7010017

3. Attia, M. (2014). Sustainability in Saudi vernacular built environment: The case of Al-Ahsa. In Vernacular Architecture: Towards a Sustainable Future (Vols. 1-0, pp. 87-92). CRC Press. https://doi.org/10.1201/b17393-18

4. Bloom, J., Blair, S. (Eds.) (2009). The Grove Encyclopedia of Islamic Art and Architecture. Oxford University Press. Retrieved 30 Aug. 2017, from http://www.oxfordreference.com/view/10.1093/acref/9780195309911.001.0001/acref-9780195309911.

5. Ettinghausen, R., Grabar, O., \& Jenkins, M. (2001). Islamic art and architecture 650-1250(2nd ed.). New Haven, CT: Yale University Press.

6. Flood, F. B. (2000). The great mosque of damascus: Studies on the makings of an ummayad visual culture. Boston: Brill.

7. Kana'an, R. (2001). Islamic art and architecture: World of art series by robert hillenbrand. london: Thames and hudson, 1999. pp. 288. price PB 8.95. 0-500-20305-9. Journal of Islamic Studies, 12(2), 235-238. doi:10.1093/jis/12.2.235

8. Mahdy, K. (n.d.). Problems facing the application of Islamic wooden artifacts ornaments in contemporary Islamic style interiors. Interna Onal Design Journal, 4(1), pp 83-98. Retrieved from http://www.journal.faadesign.com/pdf/4-1-hayam-abs.pdf

9. Petersen, A. (2002). Dictionary of islamic architecture Routledge. 